\title{
Career Options in Colon and Rectal Surgery
}

\author{
Karim Alavi, M.D., ${ }^{1}$ Robert D. Madoff, M.D., ${ }^{2}$ and David A. Rothenberger, M.D. ${ }^{2}$
}

\begin{abstract}
As Colon and Rectal Surgery has grown and diversified, the practice opportunities available have greatly expanded. The wealth of choices, may be daunting and even paralyzing for the new graduate or practitioner looking for a career change. Prior to making a decision, candidates must first make an honest assessment of their goals, abilities, and priorities. In this article, the authors briefly outline some of these challenges and help lay the groundwork for a successful decision process.
\end{abstract}

KEYWORDS: Careers, surgery, medicine, academic, practice

Objectives: Upon completion of this article, the reader should be familiar with the career options in colon and rectal surgery.

Medicine encompasses a wide variety of specialties that can be pursued in a variety of geographic and practice settings. Each individual makes his or her career decisions in a different manner. For some, the process is as simple as following in the footsteps of a parentphysician or a mentor. ${ }^{1}$ For others, the choice is more a matter of exclusion of unappealing specialties. Still others actively select a field based on specific criteria such as anticipated scope of practice, anticipated need for the specialty, geographic preference, age and type of patients, prestige, lifestyle, or income. The decision is generally influenced by one's personality, aptitude, and experiences-both good and bad-on clinical rotations in medical school or training.

Individuals choosing surgery often cite the appeal of a career that combines the management of a broad spectrum of conditions with the almost immediate gratification of intervening directly to resolve a surgical condition. The ability to make a diagnosis and provide seamless preoperative, operative, and postoperative care is highly rewarding and usually part of what motivates surgeons. Some enjoy the technical challenges, some the excitement of major trauma victims, and others are motivated by the opportunity to apply pathophysiologic principles to restore the health of critically ill patients. Other trainees are attracted to basic, translational, or clinical research, while some seek to improve technology or develop new medical devices. However, many individuals choose a surgical specialty without adequately considering the personal, academic, clinical, and financial options and implications.

General surgery applicants to colorectal training programs characterized the specialty with the following terms: "intellectually stimulating," "great diversity," "technical complexity," and "opportunities for clinical and basic research." Many cite the "friendly and collegial" atmosphere of the specialty and relate their observations that colon and rectal surgeons "took pride in caring for their patients" and did so with a level of expertise that other surgeons in their training programs did not possess.

The path from college to medical school and selection of a competitive residency and fellowship is relatively structured. Career and life-defining choices
${ }^{1}$ Division of Colon and Rectal Surgery, Department of Surgery, University of Massachusetts, Worcester, Massachusetts; ${ }^{2}$ Division of Colon and Rectal Surgery, Department of Surgery, University of Minnesota, Minneapolis, Minnesota.

Address for correspondence and reprint requests: Robert D. Madoff, M.D., Division of Colon and Rectal Surgery, Department of Surgery, University of Minnesota, 420 Delaware St. SE, MMC 450,
Minneapolis, MN 55455-0341 (e-mail: madoff@umn.edu).

Career Development; Guest Editor, David E. Beck, M.D.

Clin Colon Rectal Surg 2011;24:89-93. Copyright (C) 2011 by Thieme Medical Publishers, Inc., 333 Seventh Avenue, New York, NY 10001, USA. Tel: +1(212) 584-4662.

DOI: http://dx.doi.org/10.1055/s-0031-1278403.

ISSN 1531-0043. 
that one must make at the completion of colorectal training are all to often made with less guidance. Although the decision for some is simple ("pure" private practice, "pure" academics), for many the choice reflects a desire to create a balance between a satisfying and fulfilling clinical practice, financial gain, quality of life, academic achievement, and national and international exposure. Careers in Colon and Rectal Surgery range in scope from pure colorectal surgery (and in some cases to limited parts of colorectal surgery such as colorectal cancer or proctology) to varying degrees of general surgery and/or trauma cross-coverage to a largely general surgery practice with a colorectal "special interest." Practice setting is another important part of the equation that defines professional and personal life. Does one look towards a career in private practice, at a university, in a multispecialty clinic, or in a hybrid setting? This review explores the challenges facing today's newly trained colon and rectal surgeons as they navigate the multitude of opportunities available in this exciting field.

\section{TYPES OF PRACTICE}

In general, there are three specific practice types to choose from private practice, academic practice, and multispecialty clinic. Each has inherent advantages and disadvantages. Although this article covers generalities, each career opportunity must be viewed critically to determine its specific features that might make the job appropriate for a specific individual. Perhaps the most important advice is to "know thyself." You must honestly assess your own capabilities, motivations, and life priorities including professional, personal, and family needs. Second, is to choose associates and practice environment with care. Irrespective of practice setting, medical judgment, surgical skill, integrity, and professionalism are all important components of a successful working relationship and a satisfying career. Finally, regardless of the type of practice selected, the recent trainee/new employee will be required to sign a binding contract that will have implications on salary, benefits, lifestyle, professional advancement (both academic and practice partnership), and mobility. As described in the article on contracts in this issue, this important document is negotiable and requires careful legal review before signing.

\section{Private Practice}

Private practice, especially solo practice, offers the greatest degree of independence of the three major types of medical practice settings. Some physicians are driven to make their own business and medical decisions and do not want to have others "interfere" with how they want to run their practice. The ability to be in charge and set the standards and processes to individual specifications is appealing. However, the need to build a large referral practice can come at the cost of personal and family life, particularly if one chooses to practice alone. The individual must recognize that independence is accompanied by responsibility and financial risk. If one fails, there is no one to blame and certainly no one to come to the rescue. Furthermore, no surgeon can be truly independent in today's medical world. Hospitals have rules that must be followed, and payors have contracts and expectations for performance that must be met.

For a recent graduate, the prospect of entering private practice either alone or in a group involves multiple, frequently daunting, decisions. Most physicians have little or no training in the business aspects of running a practice so due diligence is advised. Prospective applicants often have to consider whether to join a multi- versus single- specialty practice, or whether to go solo. If one joins an existing group practice, other issues such as track to partnership, subsequent buy-in or buyout, expected income, expected call schedule ( \pm general surgery coverage), and location must be considered. Some may consider the option of purchasing an existing practice. Although this may be attractive due to the preexisting pool of patients, there are pitfalls. First, many of these patients will have had their definitive episode of care (for example, a colon resection for cancer), and any additional contact may be limited to routine follow-up or cancer surveillance or screening. In addition, a majority of these patients have built a rapport with their original surgeon; continued allegiance to the practice in the absence of that individual surgeon is not guaranteed. Some patients may just not be comfortable with a new physician fresh out of training.

The scope of the practice to join is an important variable to consider. It may seem attractive in terms of lifestyle to choose a practice limited to anorectal surgery or endoscopy, but doing so may limit future professional opportunities and job prospects. Hard-gained skills from residency and fellowship are easily lost if not maintained in practice. It is important to decide early whether to continue practicing general surgery. Although this may seem a logical aid to building one's practice, it may ultimately be counterproductive by impeding the individuals' recognition as a specialist. If one gains a reputation as an excellent general surgeon who also does colorectal surgery, it may become difficult to restrict the practice to exclusively colorectal surgery in the future. Also, many have found it is easier to become recognized as the expert in colorectal disease if specialty status is established from the outset, thus distinguishing oneself from the local general surgeons.

In private practice, one's income is usually in some way proportional to monetary production. However, individual practices apportion money differently. Overhead costs and revenues are handled variably. Some practices evenly split all income. Others pay based exclusively upon production. Many practices equally split 
a proportion of all proceeds, and divide the remainder based upon production. For example, 75\% of one's income may be divided equally among all partners, while $25 \%$ is apportioned based upon individual billing. This approach rewards those who worked harder and provides incentives for additional production. Finally, some practices award extra pay for senior partners or founding members of the group. It is important that all such matters are openly discussed, understood, and formalized in an employment agreement or contract. Specific issues include the starting salary, anticipated future salary, track to partnership (if joining a group practice), or time allotment for covering ones own income, management of overhead, decision-making processes, and call schedule obligations including cross-coverage for general surgery (if any). Other important aspects of the contract include such things as stock purchase agreement, retirement plans and their potential mobility, accounts receivable, and educational funds. Furthermore, some groups have additional avenues of income as a result of investments made, either alone or jointly, in endoscopy, surgery, or pelvic floor centers.

Finally, there are groups of practice throughout the country that have interests in academia. For instance, Colon and Rectal Surgery Associates, Ltd., is a singlespecialty, private practice that has been the focal point of the colorectal surgery residency training program at the University of Minnesota for decades. ${ }^{1}$ The partners have adjunct appointments at the University of Minnesota, but the private practice is financially independent of the University. With the colorectal surgeons at the University, they train residents, are involved in research (through the university, industry, and independently), and are active members of the American Society of Colon and Rectal Surgeons and other surgical societies. The practice commitment to the academic mission of training colorectal residents and doing research is significant, and as a result, the individual income of the partners is on average lower in comparison to other private practice groups. However, this type of practice does offer the opportunity to actively participate in research and teaching while maintaining much of the independence of a private practice.

\section{Academic Practice}

For many young surgeons considering career options, the exciting academic environment of a major teaching or research university is an ideal practice setting to achieve their goals. The interaction with medical students, surgical residents, colorectal fellows, and researchers of all disciplines is both challenging and stimulating. Typically, major research and teaching institutions serve their communities as tertiary and quaternary referral centers and as such, attract patients with complex or poorly understood problems. For some surgeons, this is the ideal patient mix. Despite reluctance for many years, many academic institutions have now established or are seeking to establish sections or divisions of colon and rectal surgery. Institutions are recruiting junior faculty who have completed a colon and rectal surgery residency at the instructor or assistant professor level. The opportunity for an academic career in colon and rectal surgery is more open than ever. On the other hand, the combination of teaching, clinical practice, and the need to be productive in the clinical, translational, or basic science research arena presents a daunting challenge.

It is important to understand the academic "tracks" available at different institutions. Typically, the tracks vary by degree of emphasis placed on patient care, teaching, clinical research, translational research, and basic research. Some tracks lead to tenure and others do not. The terminology to describe these tracks varies widely from institution to institution and one should understand the system being used before committing to a position. Common terms include adjunct, clinical or clinical scholar, and tenure-track. Typically, most academic appointments for full-time faculty are made at the assistant professor level (some may start with an appointment as an instructor). Promotion to associate professor and ultimately professor is based on achievement of specific criteria that are generally available from the institution.

Once the candidate has decided on an academic career, it is advisable to search for an institution that provides realistic support for academic achievement. If one decides to pursue a research-oriented, tenure track appointment in colon and rectal surgery, the institution should offer protected research time of at least 20 to $50 \%$ for a period of several years. The institution should also provide a research mentor, a supportive research infrastructure, and a clinical mentor for advice and assistance. Regardless of one's research focus, it is not realistic to initiate a successful academic research career without adequate protected time. In many instances, junior faculty will be provided with protected time for a set contract period (typically 3 years). When that period is up, the individual is expected to have generated adequate grant support to pay for the protected time. If there is no grant support, individuals must often increase their clinical load to support their salary. A vicious cycle can ensue: no grant, more clinical work, less time to do research, less chance of getting a grant. In many institutions, faculty appointments can be coupled with support to obtain needed additional training in biostatistics, specific research methodologies, and clinical research techniques. Ideally, other researchers in the institution can serve as collaborators and team members to help one advance in his or her research career. Potential collaborators and mentors should be interviewed to understand in detail the resources available for advancement. An 
effective clinical trials and research office with nurses and protocol writers can be invaluable.

Although it is often difficult to choose a specific area of interest fresh out of fellowship, it is important to find an area of major scientific focus early in one's academic career. Having a specific area of focus allows one to become truly expert in a given area. This expertise, in turn, optimizes the individual's chances for funding and academic success. Choosing someone with a track record in academic surgery who has a proven record of obtaining research grants, preferably in a parallel arena, will help to ensure success.

It is important to realize that success in the academic arena is defined differently from that of traditional clinical practice. Depending on the track of one's academic appointment, success may be measured primarily in terms of academic production: publications, presentations at national meetings, involvement in professional societies at the leadership level, and development of clinical programs and research protocols. For many departments, the most important measure of academic success is the ability to obtain grants, especially extramural grants. Involvement in and excellence at teaching is another important component of academic success. However, educational effort is not always rewarded commensurately with research success.

Successfully competing for grants is difficult. Usually one must "start small" and work the way up the granting ladder. The easiest grants to obtain are generally intramural start-up grants or small projects with the pharmaceutical or medical device industries. Many regional medical societies and professional societies fund research, and some direct funding specifically towards young investigators. For the colorectal surgeon, an obvious potential granting source is the Research Foundation of the American Society of Colon and Rectal Surgeons. Other professional societies that provide research grants include Society for Surgery of the Alimentary Tract (SSAT), Society of American Gastrointestinal and Endoscopic Surgeons (SAGES), American Gastroenterological Association (AGA), and Society of Surgical Oncology (SSO). Further details can be obtained at the Websites of each individual society. The hardest funding to obtain, and the most highly valued by most academic institutions, is from the $\mathrm{Na}-$ tional Institutes of Health (NIH) or other federal agencies. NIH offers a wide variety of grants, each with specific targets. For example, K-series grants are career development awards. These include K01 (mentored research scientist), K08 (mentored clinical scientist), and K23 (mentored patient-oriented research career development) grants. Many consider the most coveted NIH grant to be an R01. R01 grants are designed to support a "discrete, specified, circumscribed project to be performed by the named investigator(s) in an area representing the investigator's specific interest and competencies, based on the mission of the NIH." In practice, these grants generally require that the applicant have a successful track record of related research. Institutional support and experienced co-investigators are important components of a successful R01 application. Although basic science laboratory-based research has traditionally dominated funding, the NIH is now following a new "roadmap" to increase funding for translational and clinical researchers.

\section{Clinic Practice}

For some young surgeons, the opportunity to practice in a large clinic setting offers the best way to achieve their career goals. Some suggest that a large, multidisciplinary clinic environment can provide the best of the academic and private practice worlds. Surgical volumes tend to be high, with an often steady flow of patients needing surgery referred from the gastroenterology practices within the institution. Conversely, some surgeons object to the "corporate" decision-making that may characterize some such institutions, and others prefer a morepersonal, low-overhead, less-hectic practice pace. Major clinics place substantial value on the pursuit of research, particularly clinical research. Although expectations for publishing or obtaining grant support are less well defined than in a pure academic setting, substantial protected time may be more difficult or even impossible to come by. Finally, many clinic jobs often include appointments to the clinical or permanent faculty of an affiliated medical school.

Compensation at clinic practices varies from a negotiated salary to one based on production. The method is usually standardized throughout the institution but may change over time.

The competition for clinic positions is often intense, particularly in those institutions with substantial national and international reputations. Such institutions have far-reaching financial resources and are often recognized as clinical centers of excellence. Many have developed and maintained large clinical databases in areas such as inflammatory bowel disease and cancer. These databases greatly facilitate clinical research. In addition, the high case volumes seen at these clinics permit development of special expertise in such areas as rectal cancer, inflammatory bowel disease, and laparoscopy. Finally, because of their superb reputations, many of these centers attract research fellows as well as clinical trainees. These fellows, in addition to being helpful research collaborators, provide additional opportunities for teaching, and if they are from overseas, cultural exchange.

\section{CONCLUSION}

Colorectal surgery practice occurs in a wide variety of settings, and choosing the optimal practice involves a 
multifactorial decision process. Candidates must first make an honest assessment of their goals and priorities. Factors to consider include professional goals, abilities and training, lifestyle, family or personal needs, geographic location, opportunities for gaining professional recognition, opportunities for clinical or basic research, teaching, and financial reward. Additional derails and perspectives are provided in this issue of Clinics. Once one's aspirations and needs are clearly defined, decisions regarding the exact practice setting begin to fall into place.
DISCLOSURE

The authors have no conflicts to disclose with respect to this manuscript.

\section{REFERENCE}

1. Alavi K, Madoff RD, Rothenberger DA. Career options in colon and rectal surgery. Clin Colon Rectal Surg 2006;19(3): $114-118$ 